\title{
Expression of miR-26a exhibits a negative correlation with HMGA1 and regulates cancer progression by targeting HMGA1 in lung adenocarcinoma cells
}

\author{
NOBORU SEKIMOTO ${ }^{1}$, AYAKO SUZUKI ${ }^{2}$, YUTAKA SUZUKI $^{3}$ and SUMIO SUGANO ${ }^{1}$
}

${ }^{1}$ Department of Computational Biology and Medical Sciences, Graduate School of Frontier Sciences, The University of Tokyo, Tokyo 108-8639; ${ }^{2}$ Division of Translational Research, Exploratory Oncology Research and Clinical Trial Center, National Cancer Center, Kashiwa, Chiba 277-8577; ${ }^{3}$ Department of Computational Biology and Medical Sciences, Graduate School of Frontier Sciences, The University of Tokyo, Kashiwa, Chiba 277-8562, Japan

Received September 12, 2015; Accepted September 23, 2016

DOI: $10.3892 / \mathrm{mmr} .2016 .6053$

\begin{abstract}
Lung cancer is the most common cause of cancer-associated mortality worldwide, and the number of cases is increasing annually. Several studies have shown that microRNAs (miRNAs) control proliferation, differentiation, and apoptosis in various cell types, and increasing evidence indicates the presence of aberrant miRNA expression profiles and unique miRNA signaling pathways in several types of cancer. The present study aimed to identify miRNAs, which correlated specifically with the progression of lung cancer through the analysis of 57,100 transcripts and 1,341 small RNA expression profiles in 26 lung adenocarcinoma cell lines using next-generation sequencing. The most marked negative correlation was found between the expression of hsa-miR-26a- 1 and messenger RNA (mRNA), and a list of mRNAs, which exhibited negative correlation with hsa-miR-26a-1 were investigated. The most marked negative correlation was observed between the expression levels of hsa-miR-26a-1 and high mobility group A1 (HMGA1). Using a lung adenocarcinoma cell line, the present study analyzed the effect of the overexpression of miR-26a on the expression of HMGA1 and found that miR-26a repressed the expression of HMGA1 by reducing the mRNA levels of HMGA1. Furthermore, it was demonstrated that the overexpression of miR-26a in a lung adenocarcinoma cell line repressed cell migration, invasion and growth by targeting HMGA1. Taken together, the present study showed a significant negative correlation between the expression of miR-26a and HMGA1 in 26 lung adenocarcinoma cell lines, and
\end{abstract}

Correspondence to: Dr Sumio Sugano, Department of Computational Biology and Medical Sciences, Graduate School of Frontier Sciences, The University of Tokyo, 4-6-1 Sirokanedai, Minato-ku, Tokyo 108-8639, Japan

E-mail: ssugano@k.u-tokyo.ac.jp

Key words: microRNA-26a, lung cancer, high mobility group A1, gene expression profile, cancer progression provided evidence that the suppression of miR-26a supports the progression of cancer by stimulating the expression of HMGA1.

\section{Introduction}

Lung cancer is the most common cause of cancer-associated mortality worldwide, and the number of cases is increasing annually (1). Non-small cell lung cancer (NSCLC), including squamous cell carcinoma, adenocarcinoma and large cell carcinoma, accounts for $\sim 85 \%$ of all lung cancer types (2). Despite the increase in lung cancer survival rates as a result of improvements in diagnosis and treatment, the overall prognosis remains poor, and novel treatment strategies for patients with lung cancer are urgently required (3). An understanding of the molecular network in NSCLC is essential for the identification of effective therapeutic targets and the development of NSCLC treatment strategies; however, several aspects of the molecular network of lung carcinogenesis remain to be elucidated (4).

MicroRNAs (miRNAs) comprise a family of 21-25-nucleotide-long non-coding small RNAs, which primarily function as gene regulators. miRNAs function as repressors of translation or inducers of messenger RNA (mRNA) degradation through binding to target sites in the 3'untranslated regions (3'UTRs) of protein-coding transcripts (5). Several studies have shown that miRNAs control proliferation, differentiation and apoptosis in various cell types (6,7), and increasing evidence indicates the presence of aberrant miRNA expression profiles and unique miRNA signaling pathways in several types of cancer (8).

Our previous study comprised an integrative multi-omics analysis of 26 lung adenocarcinoma cell lines to understand how cancer harbors various genomic, epigenomic and transcriptional aberrations (9). Emerging evidence suggests that miRNAs act as oncogenic or tumor suppressor elements in tumorigenesis (10), and thus, the detection of specific miRNAs in tumor tissues or cells provides a useful diagnostic tool for presymptomatic cancer detection. Additionally, elucidation of the functions of miRNAs in tumorigenesis provides a potential novel therapeutic approach, as it may be possible to control 
the expression of miRNA by delivering synthetic pre-miRNA or antisense oligonucleotides (11). The present study aimed to identify miRNAs, which correlated specifically with the progression of lung cancer through the analysis of 57,100 transcripts and 1,341 small RNA expression profiles from 26 lung adenocarcinoma cell lines. The results identified miR-26a and HMGA1 as possible therapeutic targets and regulators of cancer progression in lung adenocarcinoma.

\section{Materials and methods}

Cell line. The H1299 human NSCLC cell line was obtained from American Type Culture Collection (Manassas, VA, USA; CRL-5803 ${ }^{\mathrm{TM}}$ ) and cultured in RPMI medium (Thermo Fisher Scientific, Inc., Waltham, MA, USA) supplemented with $10 \%$ $\mathrm{v} / \mathrm{v}$ fetal bovine serum and $1 \% \mathrm{v} / \mathrm{v}$ antibiotic solution (growth medium). The culture medium used for the other cell lines has been described elsewhere (9). The cells were incubated at $37^{\circ} \mathrm{C}$ with $5 \% \mathrm{CO}_{2}$. The other cell lines used for sequencing are listed in Table I.

Sequencing. Total RNA was extracted from the lung cancer cells using the miRNeasy Mini kit (Qiagen $\mathrm{GmbH}$, Hilden, Germany) according to the manufacturer's protocol. An mRNA library was prepared using the TruSeq RNA Sample Prep kit v2 (Illumina, Inc., San Diego, CA, USA). A small RNA library was prepared using TruSeq Small RNA Sample Prep kit (Illumina, Inc.). mRNA and small RNA sequence data were acquired using the HiSeq2500 system (Illumina, Inc.). All raw sequence data were deposited in the DNA Data Bank of Japan under the accession numbers DRA001846 (RNA-Seq) (9) and DRA003587 (small RNA-Seq).

Expression plasmids and RNA oligonucleotides. An miRNA duplex corresponding to miR26a, pcDNA3-miR26a2, was purchased from Addgene, Inc. (Cambridge, MA, USA). The pcDNA3.1 control vector used for transfection was purchased from Invitrogen; Thermo Fisher Scientific, Inc. HMGA1 small interfering RNA (siRNA) was synthesized by Sigma-Aldrich; Merck Millipore (Darmstadt, Germany). AllStars Negative Control siRNA was purchased from Qiagen $\mathrm{GmbH}$ and used as a control.

Cell transfection. The cells $\left(2.5 \times 10^{5}\right.$ cells per well) were plated in $2 \mathrm{ml}$ of growth medium in 6-well plates 1 day prior to transfection. Oligonucleotide transfection was performed using Lipofectamine 3000 (Invitrogen; Thermo Fisher Scientific, Inc.). Briefly, $7.5 \mu 1$ of Lipofectamine 3000 was diluted in $125 \mu \mathrm{l}$ of Opti-MEM1 (Gibco; Thermo Fisher Scientific, Inc.) and mixed with $125 \mu \mathrm{l}$ of DNA diluted in P3000 reagent with Opti-MEM1 medium. The mixed solutions were maintained at room temperature for $5 \mathrm{~min}$ and subsequently added to each well. The 6 -well plate was then incubated at $37^{\circ} \mathrm{C}$ with $5 \% \mathrm{CO}_{2}$ prior to use in further experiments.

Cell extraction and western blot analysis. At 3 days post-transfection, the cells were harvested and lysed in $500 \mu 1$ RIPA buffer (Wako Pure Chemical Industries, Ltd., Osaka, Japan) supplemented with $10 \%$ Complete Mini solution (Roche Diagnostics, Basel, Switzerland). The protein concentration was determined using a Pierce BCA Protein Assay kit (Thermo Fisher Scientific, Inc.). Lysate aliquots containing equal quantities of protein ( $1 \mu \mathrm{g}$ per lane) were separated by $5-20 \%$ sodium dodecyl sulfate-polyacrylamide gel electrophoresis and then electrophoretically transferred onto Immun-Blot PVDF membranes (Bio-Rad Laboratories, Inc., Hercules, CA, USA). The membranes were blocked in a solution containing $5 \%$ milk protein and $0.1 \%$ Tween 20 in Tris-buffered saline (TBS), and then incubated overnight with the appropriate primary antibody at $4^{\circ} \mathrm{C}$. The blots were then washed and incubated with an appropriate horseradish peroxidase-linked species-specific whole secondary antibody diluted 1:100,000 in TBS with $0.1 \%$ Tween 20 and $5 \%$ milk protein for $1 \mathrm{~h}$ at room temperature. Secondary antibody binding was visualized using the Las-3000 mini system (FujiFilm, Tokyo, Japan) and an ECL Prime Western Blotting detection system (GE Healthcare Life Sciences, Chalfont, UK). The following primary antibodies were purchased from Abcam (Cambridge, UK) and used for western blot analysis: Anti-tubulin (cat. no. ab4074; 1:1,000 dilution) and anti-HMGA1 (cat. no. ab129153; 1:10,000). The anti-rabbit horseradish peroxidase-linked secondary antibody (cat. no. NA934-100UL) was purchased from GE Healthcare Life Sciences.

Reverse transcription-quantitative polymerase chain reaction (RT-qPCR) analysis. Total RNA was extracted from the cells with the miRNeasy Mini kit according to the manufacturer's protocol. Primers were synthesized by Exigen (Tokyo, Japan). The miRNA expression levels were detected via SYBR Green-based real-time PCR with the mir-X miRNA qRT-PCR SYBR kit (Clontech Laboratories, Inc., Palo Alto, CA, USA) according to the manufacturer's protocol using 200-400 ng cDNA and $0.5 \mu 1100 \mathrm{nM}$ primers. The mRNA expression levels were detected with SYBR SELECT Master mix (Applied Biosystems, Inc.; Thermo Fisher Scientific, Inc.) according to the manufacturer's protocol using 400-800 ng cDNA and $2 \mu \mathrm{l}$ $100 \mathrm{nM}$ primers. The following specific primers were used for RT-qPCR: miR-26a, forward 5'-TTCAAGTAATCCAGG ATAGGCT-3' and reverse mRQ 3'primer (a component of the mir-X miRNA qRT-PCR SYBR kit); HMGA1 pair A, forward 5'-GGCCCAAATCGACCATAAAGG-3' and reverse 5'-GGA CAAATCATGGCTACCCCT-3'; HMGA1 pair B, forward 5'-CAGCGAAGTGCCAACACCTAA-3' and reverse 5'-GTT TTTGCTTCCCTTTGGTCG-3'; and U6 forward and reverse primers (provided in the mir-X miRNA qRT-PCR SYBR kit). The qPCR analysis was performed under the following conditions: $2 \mathrm{~min}$ at $50^{\circ} \mathrm{C}, 10 \mathrm{~min}$ at $95^{\circ} \mathrm{C}$, and 40 cycles at $95^{\circ} \mathrm{C}$ for $15 \mathrm{sec}$ and $60^{\circ} \mathrm{C}$ for $1 \mathrm{~min}$ on a $7900 \mathrm{HT}$ Fast Real Time PCR system (Applied Biosystems, Inc.; Thermo Fisher Scientific, Inc.). The relative expression levels of miR-26a and HMGA1 were calculated and quantified using the $\Delta \Delta \mathrm{Cq}$ method (12) following normalization to the expression levels of U6 small nuclear RNA.

Cell migration and invasion assay. The CytoSelect 96-well cell migration assay ( $8 \mu \mathrm{m}$; fluorometric format; Cell Biolabs, Inc., San Diego, CA, USA) and CytoSelect 96-well cell invasion assay basement membrane (fluorometric format; Cell Biolabs, Inc.) were used to analyze cell migration and invasion 
activities, respectively. Equal numbers of cells $\left(1.0 \times 10^{5}\right.$ cells per chamber), which had been transfected with RNA duplex or plasmid for $48 \mathrm{~h}$, were seeded into the upper chambers containing serum-free medium. Medium with $10 \%$ serum was added to the lower chambers as a chemoattractant. After $12 \mathrm{~h}$ incubation at $37^{\circ} \mathrm{C}, 4 \mathrm{X}$ lysis buffer/CyQuant GR dye solution (Cell Biolabs, Inc.) was added to the lower chambers. The numbers of migrated and invaded cells were counted via fluorescence detection at 480/520 nm, using a FLUOstar OPTIMA microplate reader (BMG Labtech, GmbH, Ortenberg, Germany).

Cell proliferation assay. The cells were counted using a Cell Counting Kit-8 (CCK-8; Dojindo Molecular Technologies, Inc., Kumamoto, Japan). In brief, $10 \mu 1$ of CCK-8 solution was added to the cells in each well of a 96-well plate, followed by incubation for $2 \mathrm{~h}$ at $37^{\circ} \mathrm{C}$ in $5 \% \mathrm{CO}_{2}$. The solution absorbance was measured at $450 \mathrm{~nm}$ in an iMark microplate absorbance reader (Bio-Rad Laboratories, Inc.).

Luciferase assays. To construct the luciferase reporter vectors, a 3'untranslated region (UTR) fragment of wild-type (WT) human HMGA1, which contained miR-26a binding sites, was amplified from genomic DNA using the following primers: Forward 5'-TCGAAAGCTTCCCAAATCGACCATAAA GGGTG-3' and reverse 5'-TCGAACTAGTTCCAGAAA AGGATATTTTTTTTATTCAAG-3'. The amplification was performed with Phusion High-Fidelity DNA Polymerase (New England BioLabs, Inc., Ipswich, MA, USA) under the following conditions: $30 \mathrm{sec}$ at $98^{\circ} \mathrm{C}$; and 30 cycles at $98^{\circ} \mathrm{C}$ for $10 \mathrm{sec}, 65^{\circ} \mathrm{C}$ for $30 \mathrm{sec}$ and $72^{\circ} \mathrm{C}$ for $90 \mathrm{sec}$, followed by $72^{\circ} \mathrm{C}$ for $10 \mathrm{~min}$; on a GeneAmp PCR System 9700 (Applied Biosystems; Thermo Fisher Scientific, Inc.). The amplified fragment was inserted into the pMIR-REPORT miRNA expression reporter vector (Applied Biosystems, Inc., Thermo Fisher Scientific, Inc.) at the HindIII/SpeI sites. Mutations were generated in the miR-26a binding sites using the KOD-Plus-Mutagenesis kit (Toyobo Co., Ltd., Osaka, Japan) and the following primers: Forward 5'-ATGAACTATAAA AAAAATATCCTTTTCTGGAA-3' and reverse 5'-CTG CAAATAGGAAACCAGAGAGGG-3'. The sequences of the resulting plasmids containing the WT or mutated (Mut) 3'UTR of HMGA1 were verified by DNA sequencing. The Renilla luciferase-encoding plasmid, pRL-TK (Promega Corporation, Madison, WI, USA) was used as an internal control. The H1299 cells $\left(1.2 \times 10^{3}\right.$ cells per well) were plated in $100 \mu \mathrm{l}$ of growth medium in 96-well plates one day prior to transfection. At room temperature, the H1299 cells were co-transfected with either the miR-26a expression vector or the control vector along with the pRL-TK vector and pMIR-REPORT miRNA, the expression reporter vector containing the WT or Mut 3'UTR of HMGA1. After $48 \mathrm{~h}$, the cells were harvested and subjected to luciferase analysis using the Dual-Luciferase reporter assay system (Promega Corporation) in a Centro LB 960 (Berthold Technologies, Bad Wildbad, Germany).

Statistical analysis. The sequence data were mapped to the human reference genome, hg19, in the UCSC genome browser (https://genome.ucsc.edu) using the Efficient Large-Scale Alignment of Nucleotide Databases tool (Illumina, Inc.), as
Table I. Cancer cell lines included for assessment in the present study.

\begin{tabular}{lll}
\hline Cell line & Distributor & Cat. no. \\
\hline A427 & ATCC & HTB-53 \\
A549 & ATCC & CCL-185 \\
H322 & ATCC & CRL-5806 \\
H1299 & ATCC & CRL-5803 \\
H1437 & ATCC & CRL-5872 \\
H1648 & ATCC & CRL-5882 \\
H1650 & ATCC & CRL-5883 \\
H1703 & ATCC & CRL-5889 \\
H1819 & ATCC & CRL-5897 \\
H1975 & ATCC & CRL-5908 \\
H2126 & ATCC & CCL-256 \\
H2228 & ATCC & CRL-5935 \\
H2347 & ATCC & CRL-5942 \\
RERF-LC-Ad1 & JCRB & JCRB1020 \\
RERF-LC-Ad2 & JCRB & JCRB1021 \\
RERF-LC-MS & JCRB & JCRB0081 \\
RERF-LC-OK & JCRB & JCRB0811 \\
VMRC-LCD & JCRB & JCRB0814 \\
ABC-1 & JCRB & JCRB0815 \\
PC-3 & JCRB & JCRB0077 \\
PC-9 & RIKEN BRC & RCB4455 \\
RERF-LC-KJ & RIKEN BRC & RCB1313 \\
LC2/ad & RIKEN BRC & RCB0440 \\
II-18 & RIKEN BRC & RCB2093 \\
PC-7 & IBL & Upon request \\
PC-14 & IBL & Upon request \\
\hline
\end{tabular}

ATCC, American Type Culture Collection (http://www.atcc.org/); JCRB, Japanese Collection of Research Bioresources (Osaka, Japan); RIKEN BRC, RIKEN BioResource Center (Ibaraki, Japan); IBL, Immunobiological Laboratories (Gunma, Japan).

described in a previous study (9). Using perl script, the parts per million $(\mathrm{ppm})$ and reads per kilobase per million were calculated as miRNA and mRNA expression levels, respectively. Correlation coefficients were calculated using Pearson product-moment correlation. A heat map was generated using heatmap. 2 in the gplots of R.

\section{Results}

Negative correlation between the expression levels of miR-26a and HMGAl in 26 human lung adenocarcinoma cell lines. The present study assessed small RNAs in 26 human lung adenocarcinoma cell lines (Table I) using next-generation sequencing. A total of 1,341 miRNAs were selected from these data, and their expression levels were calculated in ppm. To determine the associations between miRNA and mRNA expression throughout the genome, the correlation coefficient was calculated between each miRNA and individual mRNAs using mRNA data from a previous study (9). A heat map of these correlation coefficients is shown in Fig. 1A. As shown 
in the histogram in Fig. 1A, a higher number of mRNAs were negatively correlated with miRNAs, compared with the number of mRNAs positively correlated with miRNAs. This suggested the predominantly repressive effects of miRNAs on mRNAs in these cell lines. Among the miRNAs, hsa-miR-26a-1, the major precursor RNA of miR-26a, exhibited the most marked negative correlation with overall mRNA expression (the 10 miRNAs most negatively correlated with overall mRNA expression are shown in Table II), suggesting that miR-26a may have the most marked effect within these adenocarcinoma cell lines. Subsequently, the present study aimed to identify the mRNA correlated most significantly with hsa-miR-26a-1. It was found that the HMGA1 mRNA exhibited the most marked negative correlation with hsa-miR-26a-1, suggesting that it was affected most by miR-26a. HMGA1 is a master chromatin structural regulator, which primarily mediates its gene regulatory activity by interacting directly with A/T-rich DNA sequences located in promoter and enhancer regions (13). HMGA1 also exhibited a negative correlation with another miR-26a precursor, hsa-miR-26a-2 (Fig. 1B and Table III).

miR-26a silences the expression of HMGAl in the H1299 human lung adenocarcinoma cell line by degrading HMGAI $m R N A$. To determine the functionality of the negative correlation between miR-26a and HMGA1, miR-26a was overexpressed in the H1299 NSCLC cell line, which has low and high endogenous levels of miR-26a and HMGA1, respectively. The expression level of miR-26a was increased $>500$-fold following transfection with the miR-26a expression vector (Fig. 2A). This led to reduced protein levels of HMGA1, as shown in Fig. 2B. The mRNA level of HMGA1 also decreased in response to the overexpression of miR-26a (Fig. 2C). Thus, miR-26a appeared to suppress the protein expression of HMGA1 by reducing the mRNA level of HMGA1.

To examine whether HMGA1 is a direct target of miR-26a, the present study performed a luciferase reporter assay using a plasmid construct containing the 3'UTR of HMGA1 mRNA. The 3'UTR contained putative miR-26a binding sites, and a reporter plasmid containing mutated miR-26a binding sites was used as a control (Fig. 3A). These reporter constructs, containing either miR-26a or a control expression vector, were co-transfected into H1299 cells. The luciferase activity of the reporter harboring the HMGA1 WT 3'UTR was reduced by $50 \%$ following co-transfection with the miR-26a expression vector. By contrast, the activity of the Mut construct was not altered by co-transfection with miR-26a. These results suggested that HMGA1 mRNA is a direct target of miR-26a (Fig. 3B).

miR-26a represses migration, invasion and growth in H1299 cells. To investigate the role of miR-26a in the capacities of cells to metastasize and invade, miR-26a was overexpressed in H1299 cells and the resulting cellular phenotype was evaluated. In the cells overexpressing miR-26a, 60\% reductions were observed in the migration and invasion capacities, compared with the control (Fig. 4A). These data suggested that miR-26a suppressed migration and invasiveness in H1299 cells. Furthermore, reduced cell growth was observed in response to the overexpression of miR-26a, compared with the control, which suggested that miR-26a repressed the proliferation of the H1299 cells (Fig. 4B).

HMGAl promotes cell migration, invasion and cell growth in H1299 cells. To identify cellular phenotypes, which varied with altered expression of HMGA1, cell invasion and migration activity assays were performed. HMGA1 was knocked down using siRNA and the protein expression of HMGA1 was successfully suppressed by $80 \%$ (Fig. 5A). Upon HMGA1 knockdown, a $60 \%$ decrease in migration capacity and $50 \%$ decrease in invasiveness were observed, compared with the control (Fig. 5B). These data indicated that a high expression level of HMGA1 enhanced cell migration and invasiveness in H1299 cells. Furthermore, a reduction in the rate of cell growth was observed following HMGA1 knockdown, suggesting a positive role of HMGA1 in the promotion of H1299 cell growth (Fig. 5C).

\section{Discussion}

In the present study, miRNA expression levels were analyzed in 26 human lung adenocarcinoma cell lines, and these were compared against mRNA data from our previous study (9). It was found that miR-26a exhibited the most marked negative correlation with overall mRNA expression levels. Furthermore, it was found that HMGA1 mRNA exhibited the most marked negative correlation with miR-26a. Although miR-26a and HMGA1 were selected from statistical analysis, further analyses confirmed that these molecules affected the migration, invasion and growth properties of a human adenocarcinoma cell line. These results were consistent with previous studies, in which miR-26a was identified as a tumor suppressor $(14,15)$ and HMGA1 as an oncoprotein (16) in various cell types. Accordingly, a combined approach, which evaluates cell lines using miRNA-seq may assist in identifying miRNAs, which are biologically relevant in particular cancer cell types.

After completion of the present study, it came to our attention that RERF-LC-OK cells are mis-identified and are actually Marcus human astrocytoma cells. The correlation coefficients were recalculated using 25 cell lines without RERF-LC-OK. The correlation coefficient between overall gene expression and hsa-miR-26a-1 changed from -0.1105 to -0.1116 and therefore remains the most negatively related among miRs. The correlation coefficient between HMGA1 and hsa-miR-26a-1 changed from -0.4927 to -0.4830 , becoming the fifth negatively correlated gene rather than the first, but still highly correlated. The correlation coefficient between HMGA1 and hsa-miR-26a-2 changed from -0.3758 to -0.3499 .

Previous studies have reported the presence of other miR-26a targets, including phosphatase and tensin homolog (PTEN) and enhancer of zeste homolog 2 (EZH2), in lung cancer cells $(17,18)$. As PTEN is a known tumor suppressor (19), it was suggested that reduced expression of miR-26a in lung cancer cells suppresses the carcinomatous phenotype. This is inconsistent with the experimental data obtained in the present study, resulting from the overexpression of miR-26a in a lung adenocarcinoma cell line. This 
A
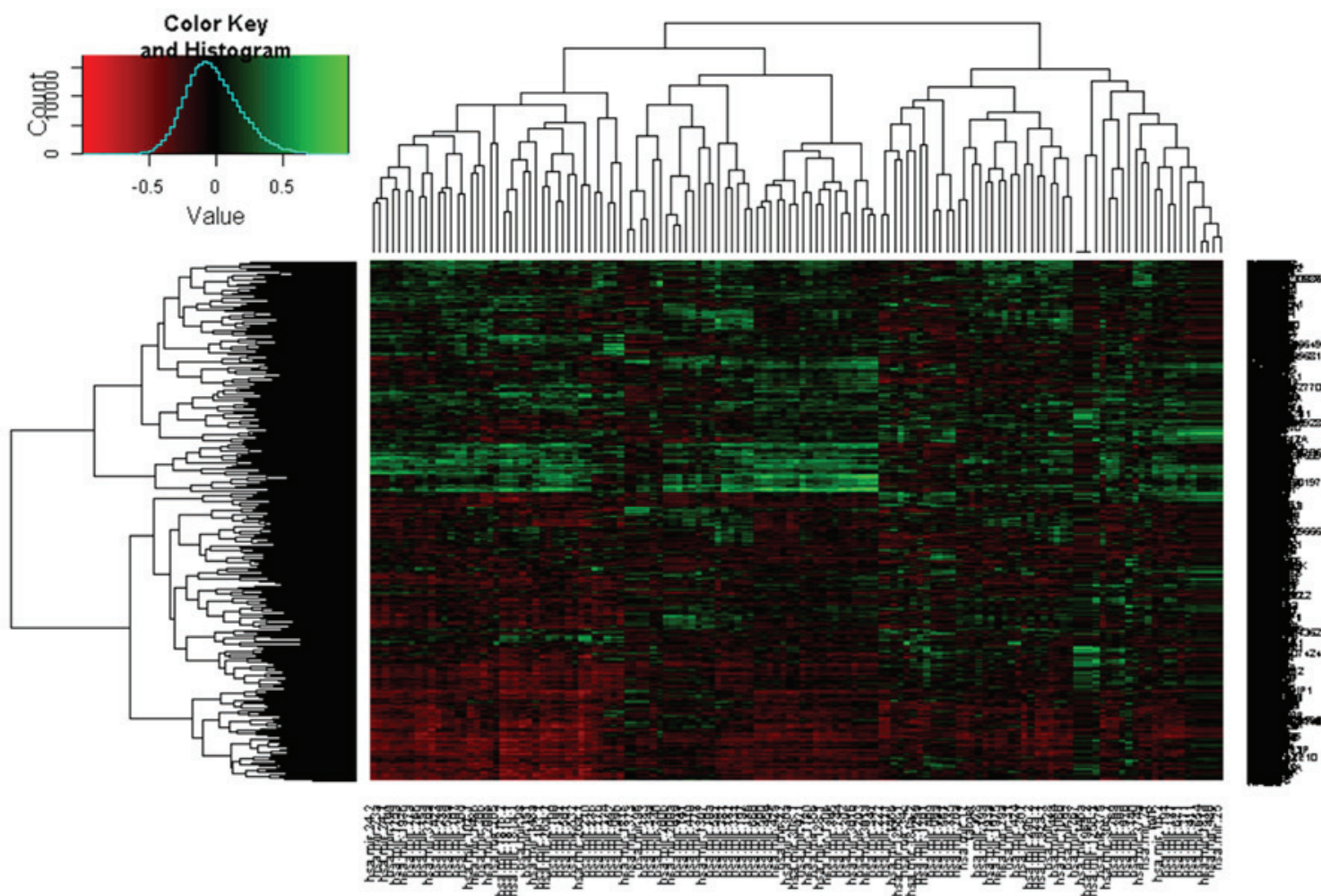

B
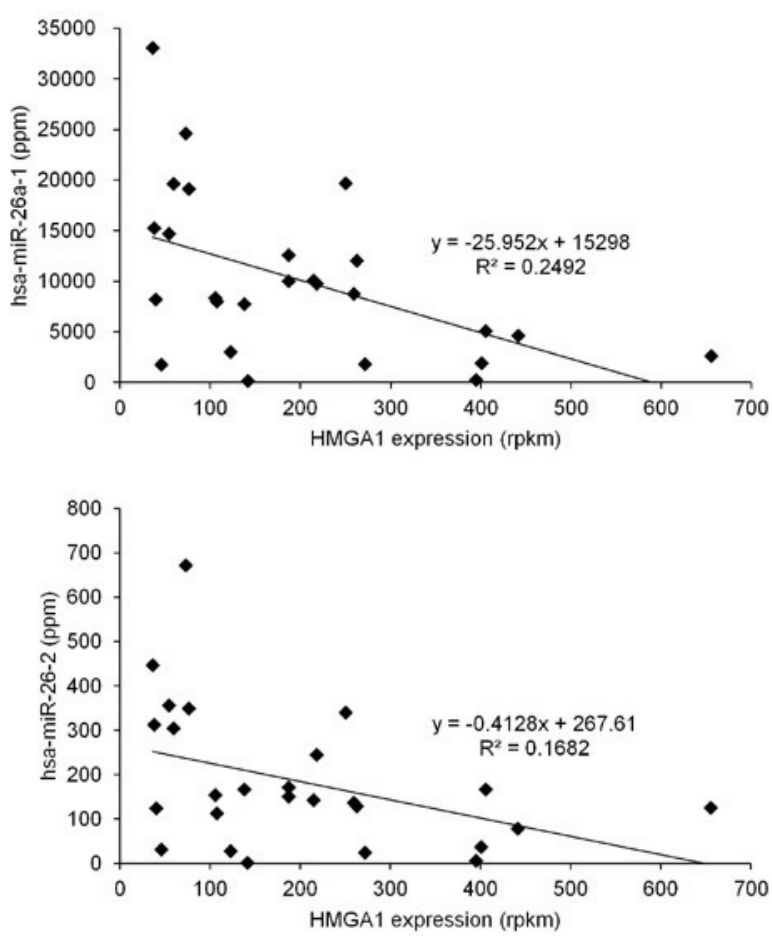

Figure 1. Expression profiles of miR-26a and HMGA1 in 26 human lung adenocarcinoma cell lines. (A) Heat map showing the correlation coefficients between the miRNA and mRNA expression levels in the 26 human lung adenocarcinoma cell lines. The vertical axis represents each mRNA, and the horizontal axis represents each miRNA. The mRNA and miRNA expression levels were calculated in rpkm and ppm, respectively. The mRNA and miRNA expression levels in this heat map exceeded $40 \mathrm{rpkm}$ and $100 \mathrm{ppm}$, respectively. (B) Scatter plots of the expression levels of HMGA1 and miR-26a in the 26 human lung adenocarcinoma cell lines. A negative correlation was observed in these 26 human lung adenocarcinoma cell lines. Detailed expression levels are listed in Table IV. HMGA1, high mobility group A1; miR, microRNA; rpkm, reads per kilobase per million; ppm, parts per million.

discrepancy may be attributable to the different cell lines used in the assay. No marked correlation between miR-26a and the mRNA expression of PTEN were found in the 26 human lung adenocarcinoma cell lines assessed in the present study. By contrast, HMGA1 exhibited the most marked correlation with miR-26a. Furthermore, the original expression level PTEN in the H1299 cells was 50-fold lower, compared with that of HMGA1. Therefore, the effects of the overexpression of 
Table II. Top 10 miRNAs exhibiting negative correlation with overall mRNA expression.

\begin{tabular}{clc}
\hline Rank & \multicolumn{1}{c}{ miRNA } & Correlation coefficient \\
\hline 1 & hsa-miR-26a-1 & -0.1105 \\
2 & hsa-miR-24-2 & -0.0861 \\
3 & hsa-miR-210 & -0.0859 \\
4 & hsa-miR-23b & -0.0790 \\
5 & hsa-miR-320a & -0.0754 \\
6 & hsa-miR-24-1 & -0.0728 \\
7 & hsa-miR-181b-2 & -0.0705 \\
8 & hsa-miR-26b & -0.0654 \\
9 & hsa-miR-501 & -0.0603 \\
10 & hsa-miR-98 & -0.0588
\end{tabular}

Correlation coefficient represents the average of all correlation coefficients between the expression of an miRNA and all mRNA. miR, microRNA.

Table III. Top 10 mRNAs exhibiting the highest negative correlations with the expression of hsa-miR-26a-1.

\begin{tabular}{lcc}
\hline mRNA & $\begin{array}{c}\text { hsa-miR-26a-1 } \\
\text { (correlation coefficient) }\end{array}$ & $\begin{array}{c}\text { hsa-miR-26a-2 } \\
\text { (correlation coefficient) }\end{array}$ \\
\hline HMGA1 & -0.4927 & -0.3758 \\
SRSF3 & -0.4845 & -0.3640 \\
PRDX3 & -0.4844 & -0.3977 \\
COPS6 & -0.4755 & -0.3022 \\
TMEM222 & -0.4720 & -0.3032 \\
MCL1 & -0.4546 & -0.4291 \\
MAP2K2 & -0.3810 & -0.2176 \\
MATR3 & -0.3426 & -0.1367 \\
EIF5A & -0.3150 & -0.1595 \\
CKS2 & -0.3100 & -0.1851 \\
\hline
\end{tabular}

All 10 mRNAs exhibited sequence complementarity with miR-26a. miR, microRNA.

miR-26a on PTEN may not significantly affect the cellular phenotype of this cell line.

In the present study, a negative correlation was demonstrated between EZH2 mRNA, which is involved in cancer progression (20), and miR-26a (data not shown) in the 26 evaluated cell lines. However, it was noted that the expression levels of EZH2 were also low in these cell lines, being 10-fold lower than the expression levels of HMGA1 in the H1299 cells. This finding suggested that EZH2 may have a minimal effect on the cellular phenotype, compared with HMGA1.

Although several studies have demonstrated the importance of the miR-26a/HMGA1 pair in bladder and breast cancer $(21,22)$, the present study is the first, to the best of our knowledge, to describe the role of this pair in lung adenocarcinoma, and the first to demonstrate a correlation between the expression of miR-26a
A

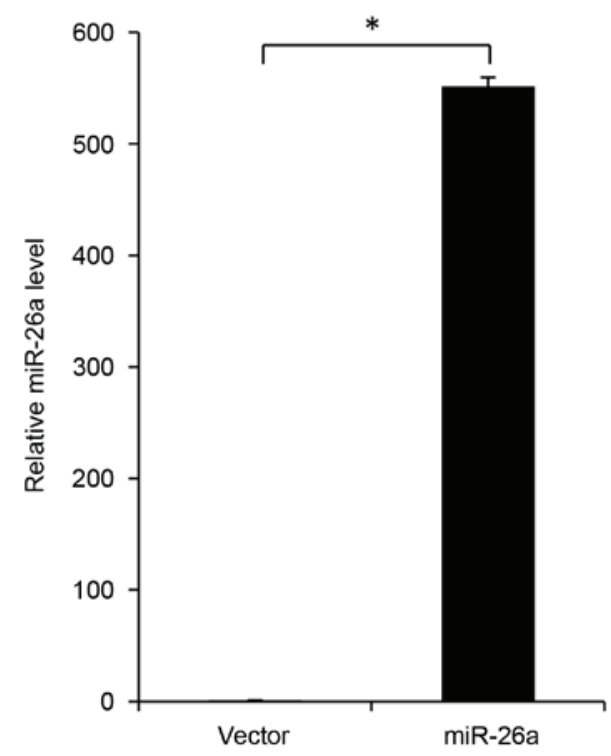

B
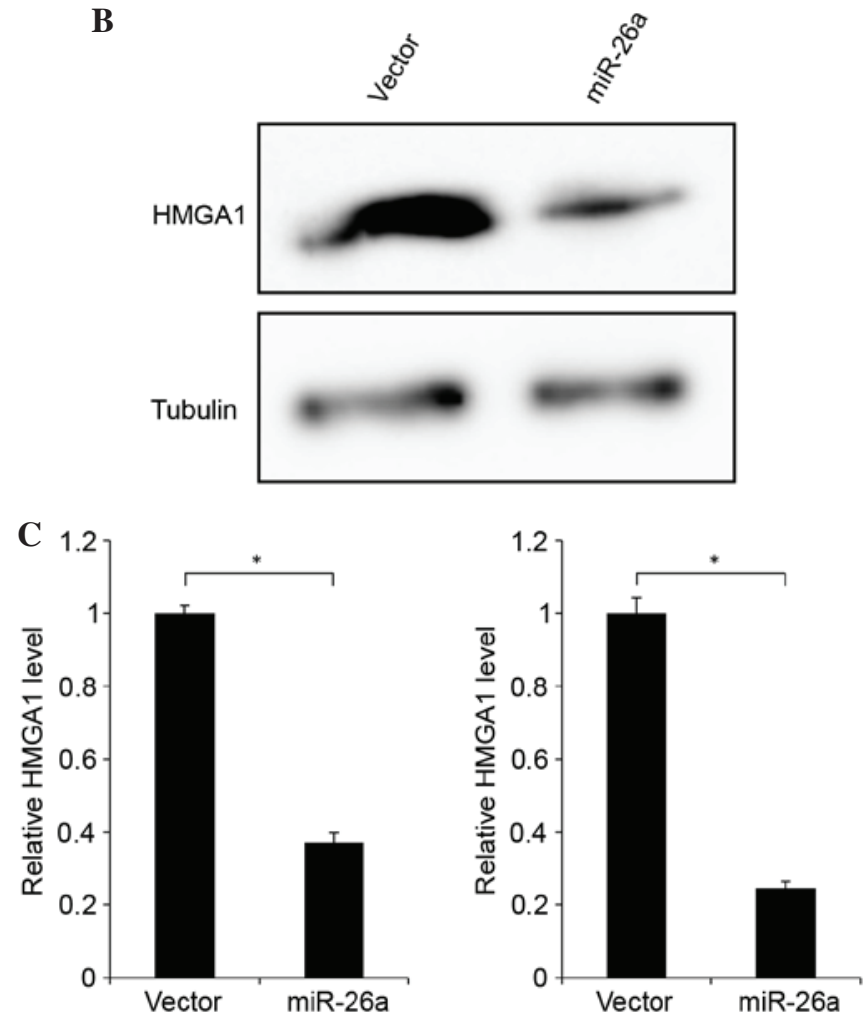

Figure 2. Overexpression of miR-26a reduces the expression of HMGA1 in H1299 cells. (A) RT-qPCR analysis indicated increased expression of miR-26a following transfection with the miR-26a expression vector, compared with the control H1299 cells ("P<0.05). (B) Western blot analysis of the protein expression levels of HMGA1 in H1299 cells transfected with the miR-26a expression vector or control vector. (C) RT-qPCR analysis of the mRNA expression levels of HMGA1 in H1299 cells transfected with the miR-26a expression vector or control vector. Primer pairs A (left) and B (right) were used to generate the data in the two bar graphs. Values are presented as the mean \pm standard deviation $(" \mathrm{P}<0.05)$. HMGA1, high mobility group A1; miR, microRNA; RT-qPCR, reverse transcription-quantitative polymerase chain reaction.

and HMGA1, as previous reports focused on either the expression of miR-26a or HMGA1 in cancer cell lines. The results of the present study supported those of previous reports, which demonstrated downregulated expression of the hsa-miR-26a-1 
Table IV. Expression profiles of miR-26a and HMGA1 in 26 human lung adenocarcinoma cell lines.

\begin{tabular}{|c|c|c|c|}
\hline Cell line & HMGA1 (rpkm) & hsa-miR-26a-1 (ppm) & hsa-miR-26a-2 (ppm) \\
\hline $\mathrm{LC} 2 / \mathrm{ad}$ & 259.46 & 8783.11 & 136.66 \\
\hline PC-9 & 214.60 & 10064.79 & 143.15 \\
\hline PC-14 & 271.86 & 1839.67 & 24.69 \\
\hline RERF-LC-Ad1 & 187.24 & 12611.13 & 150.3 \\
\hline RERF-LC-Ad2 & 36.35 & 33088.83 & 446.77 \\
\hline RERF-LC-KJ & 40.06 & 8210.37 & 124.60 \\
\hline PC-3 & 394.92 & 236.21 & 6.22 \\
\hline II-18 & 218.35 & 9760.12 & 245.16 \\
\hline PC-7 & 141.68 & 150.08 & 1.88 \\
\hline RERF-LC-MS & 59.37 & 19648.70 & 305.18 \\
\hline RERF-LC-OK & 54.30 & 14726.07 & 355.79 \\
\hline VMRC-LCD & 122.73 & 3042.91 & 27.86 \\
\hline ABC-1 & 105.58 & 8365.73 & 154.68 \\
\hline A549 & 137.85 & 7758.11 & 166.68 \\
\hline H1650 & 38.17 & 15278.56 & 313.01 \\
\hline A427 & 263.01 & 12057.88 & 128.49 \\
\hline H322 & 76.69 & 19137.18 & 349.67 \\
\hline H1437 & 107.39 & 7985.98 & 112.45 \\
\hline H1648 & 250.44 & 19675.11 & 340.35 \\
\hline H1975 & 186.94 & 10008.00 & 171.78 \\
\hline H2126 & 45.77 & 1745.25 & 31.36 \\
\hline H1299 & 400.87 & 1896.35 & 37.03 \\
\hline H2228 & 655.55 & 2634.11 & 125.04 \\
\hline H2347 & 441.60 & 4622.47 & 78.27 \\
\hline H1703 & 405.61 & 5101.17 & 166.53 \\
\hline H1819 & 73.21 & 24630.17 & 672.00 \\
\hline
\end{tabular}

HMGA1, high mobility group A1; miR, microRNA; rpkm, reads per kilobase per million; ppm, parts per million.


Figure 3. Validation of HMGA1 as a direct target of miR-26a. (A) Putative miR-26a binding sites in the 3'UTR of HMGA1. (B) H1299 cells were co-transfected with either an miR-26a expression vector (black bar) or control vector (white bar) along with the pRL-TK vector and pMIR-REPORT miRNA expression reporter vector containing the WT or Mut 3'UTR of HMGA1. The relative luciferase activities were measured $48 \mathrm{~h}$ post-transfection and normalized to Renilla luciferase activity ( $\left.{ }^{*} \mathrm{P}<0.05\right)$. WT, wild-type; Mut, mutant; 3'UTR, 3'untranslated region; HMGA1, high mobility group A1; miR, microRNA. 
A
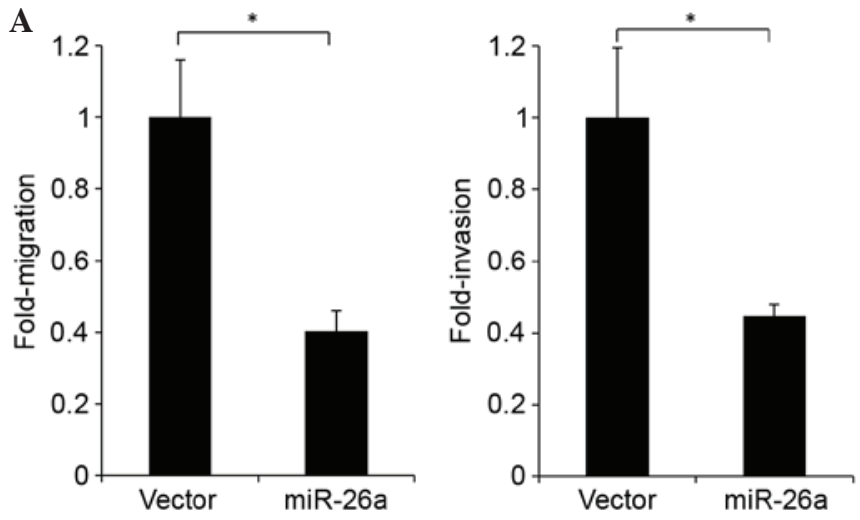

B

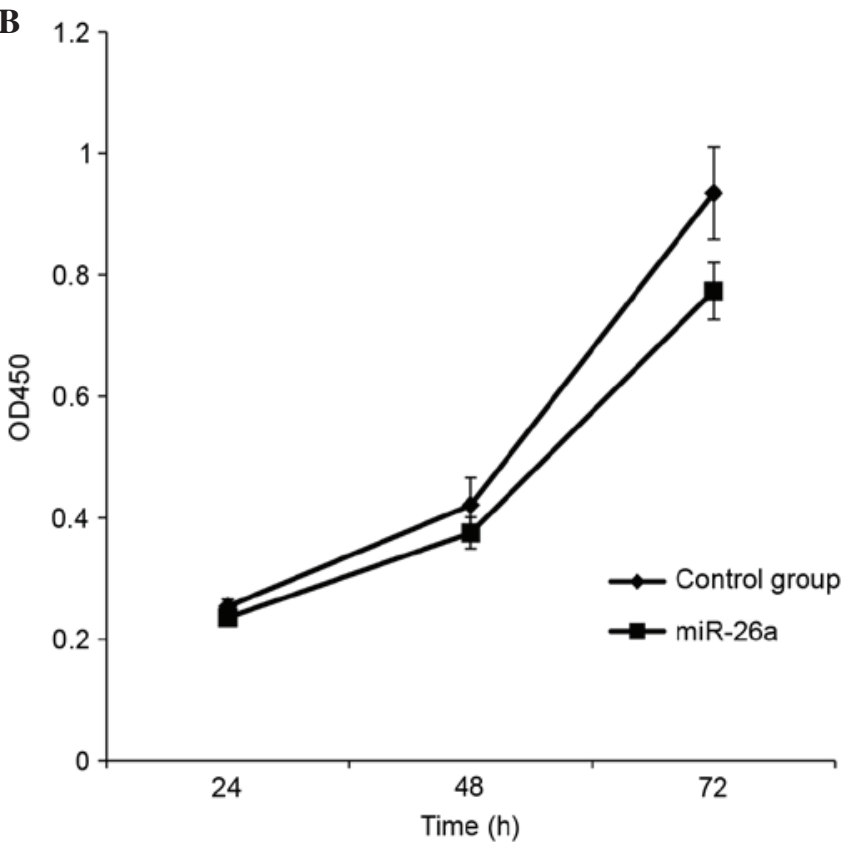

Figure 4. Overexpression of miR-26a reduces cell invasion and migration activities and reduces the growth rate of $\mathrm{H} 1299$ cells. (A) Cell invasion and migration capacities were measured in $\mathrm{H} 1299$ cells transfected with an miR-26a expression vector or control vector ("P<0.05). (B) Cell proliferation was examined. Data are presented as the mean OD at $450 \mathrm{~nm} \pm$ standard deviation at 24, 48 and $72 \mathrm{~h}$ post-vector transfection. miR, microRNA; OD, optical density.

precursor (23) and upregulated expression of HMGA1 (24) in lung cancer, compared with corresponding normal tissues. Additionally, the present study demonstrated that miR-26a may have directly silenced the expression of HMGA1, and thus affected the migration and invasion potential of H1299 lung adenocarcinoma cell lines through HMGA1. These findings are consistent with those of a previous report, in which HMGA1 silencing reduced the metastatic potential of pancreatic adenocarcinoma (25). The present study also observed that a reduction in the expression of HMGA1 led to a reduced growth rate in H1299 cells. The suppression of cell growth in response to the suppressed expression of HMGA1 has also been observed in breast cancer and osteogenic sarcoma (26).

Taken together, the low level of miR-26a in lung adenocarcinoma cell lines may result in a high level of HMGA1, thus supporting cancer progression. It is possible that, in the future, restoration of the expression of miR-26a may offer a novel strategy for the treatment of lung adenocarcinoma.

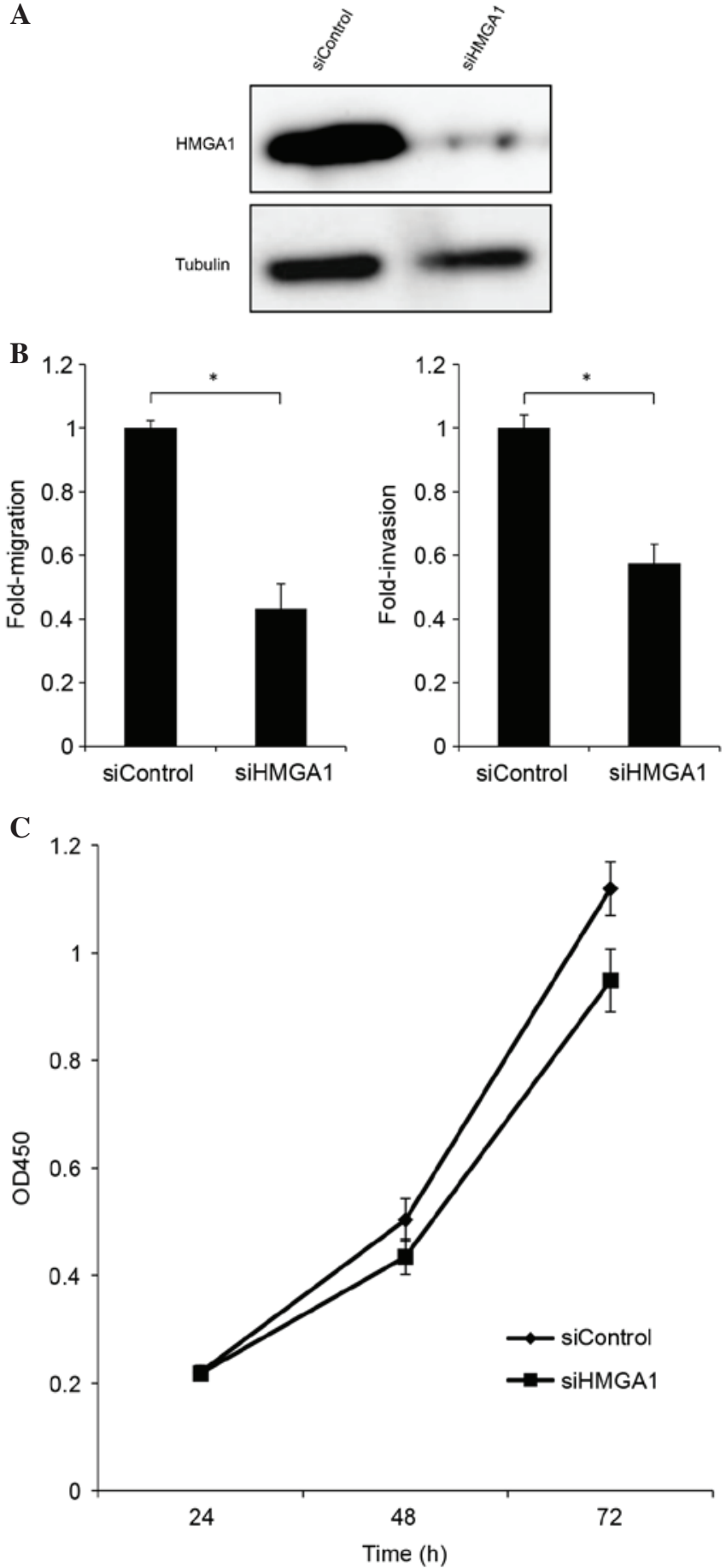

Figure 5. HMGA1 knockdown reduces cell invasion and migration activity, and reduces growth rate in $\mathrm{H} 1299$ cells, similar to the overexperssion of miR-26a. (A) Representative western blots of the expression of HMGA1 and tubulin $72 \mathrm{~h}$ post-transfection. (B) Cell invasion and migration capacities were measured in the cells with HMGA1 knocked down or control-siRNA transfected H1299 cells ("P<0.05). (C) Cell proliferation was examined. Data are presented as the mean ODs at $450 \mathrm{~nm} \pm$ standard deviations at 24,48 and $72 \mathrm{~h}$ post-siRNA transfection. HMGA1, high mobility group A1; miR, microRNA; si, small interfering RNA; OD, optical density.

\section{Acknowledgements}

This study was supported by a Grant-in-Aid for Scientific Research in the Priority Area 'Genome Science' (grant no. 221S0002) from the Ministry of Education, Culture, 
Sports, Science and Technology of Japan. This manuscript was proofread by the Enago English language editing service (https://www.enago.jp/).

\section{References}

1. Pirozynski M: 100 years of lung cancer. Respir Med 100 2073-2084, 2006.

2. D'Addario G,Früh M,Reck M,Baumann P,Klepetko W and Felip E; ESMO Guidelines Working Group: Metastatic non-small-cell lung cancer: ESMO clinical practice guidelines for diagnosis, treatment and follow-up. Ann Oncol 21 (Suppl 5): v116-v119, 2010.

3. Saintigny P and Burger JA: Recent advances in non-small cell lung cancer biology and clinical management. Discov Med 13: 287-297, 2012

4. Liloglou T, Bediaga NG, Brown BR, Field JK and Davies MP: Epigenetic biomarkers in lung cancer. Cancer Lett 342: 200-212, 2014.

5. Zhang W, Dahlberg JE and Tam W: MicroRNAs in tumorigenesis: A primer. Am J Pathol 171: 728-738, 2007.

6. Wu F, Yang Z and Li G: Role of specific microRNAs for endothelial function and angiogenesis. Biochem Biophysi Res Comm 386: 549-553, 2009.

7. He L and Hannon GJ: MicroRNAs: Small RNAs with a big role in gene regulation. Nat Rev Genet 5: 522-531, 2004.

8. Wang V and $\mathrm{Wu} \mathrm{W}$ : MicroRNA-based therapeutics for cancer. BioDrugs 23: 15-23, 2009.

9. Suzuki A, Makinoshima H, Wakaguri H, Esumi H, Sugano S, Kohno T, Tsuchihara K and Suzuki Y: Aberrant transcriptional regulations in cancers: Genome, transcriptome and epigenome analysis of lung adenocarcinoma cell lines. Nucleic Acids Res 42 : $13557-13572,2014$.

10. Feng Z, Zhang C, Wu R and $\mathrm{Hu} \mathrm{W}$ : Tumor suppressor p53 meets microRNAs. J Mol Cell Biol 3: 44-50, 2011.

11. Gulino R, Forte S, Parenti R, Memeo L and Gulisano M: MicroRNA and pediatric tumors: Future perspectives. Acta Histochem 117: 339-354, 2015

12. Livak KJ and Schmittgen TD: Analysis of relative gene expression data using real-time quantitative PCR and the 2(-Delta Delta C(T)) Method. Methods 25: 402-408, 2001.

13. Benecke AG and Eilebrecht S: RNA-Mediated regulation of HMGA1 function. Biomolecules 5: 943-957, 2015.

14. Zeitels LR, Acharya A, Shi G, Chivukula D, Chivukula RR, Anandam JL, Abdelnaby AA, Balch GC, Mansour JC, Yopp AC, et al: Tumor suppression by miR-26 overrides potential oncogenic activity in intestinal tumorigenesis. Genes Dev 28 : 2585-2590, 2014.
15. Zhao S, Ye X, Xiao L, Lian X, Feng Y, Li F and Li L: MiR-26a inhibits prostate cancer progression by repression of Wnt5a. Tumour Biol 35: 9725-9733, 2014.

16. Pierantoni GM, Rinaldo C, Esposito F, Mottolese M, Soddu S and Fusco A: High mobility group A1 (HMGA1) proteins interact with p53 and inhibit its apoptotic activity. Cell Death Differ 13: 1554-1563, 2006

17. Liu B, Wu X, Liu B, Wang C, Liu Y, Zhou Q and Xu K: MiR-26a enhances metastasis potential of lung cancer cells via AKT pathway by targeting PTEN. Biochim Biophysica Acta 1822: 1692-1704, 2012.

18. Dang X, Ma A, Yang L, Hu H, Zhu B, Shang D, Chen T and Luo Y: MicroRNA-26a regulates tumorigenic properties of EZH2 in human lung carcinoma cells. Cancer Genet 205: 113-123, 2012.

19. Li J, Yen C, Liaw D, Podsypanina K, Bose S, Wang SI, Puc J, Miliaresis C, Rodgers L, McCombie R, et al: PTEN, a putative protein tyrosine phosphatase gene mutated in human brain, breast, and prostate cancer. Science 275: 1943-1947, 1997.

20. Varambally S, Dhanasekaran SM, Zhou M, Barrette TR, Kumar-Sinha C, Sanda MG, Ghosh D, Pienta KJ, Sewalt RG, Otte AP, et al: The polycomb group protein EZH2 is involved in progression of prostate cancer. Nature 419: 624-629, 2002

21. Lin Y, Chen H, Hu Z, Mao Y, Xu X, Zhu Y, Xu X, Wu J, Li S, Mao Q, et al: miR-26a inhibits proliferation and motility in bladder cancer by targeting HMGA1. FEBS Lett 587: 2467-2473, 2013.

22. Zhao XX, Yuan QZ, Mu DP, Sun DW, Bo QA, Pan GZ, Li GQ, Cui T, Ding PP, You FP, et al: MicroRNA-26a inhibits proliferation by targeting high mobility group AT-hook 1 in breast cancer. Int J Clin Exp Pathol 8: 368-373, 2015.

23. Yanaihara N, Caplen N, Bowman E, Seike M, Kumamoto K, Yi M, Stephens RM, Okamoto A, Yokota J, Tanaka T, et al: Unique microRNA molecular profiles in lung cancer diagnosis and prognosis. Cancer Cell 9: 189-198, 2006.

24. Kettunen E, Anttila S, Seppänen JK, Karjalainen A, Edgren H, Lindström I, Salovaara R, Nissén AM, Salo J, Mattson K, et al: Differentially expressed genes in nonsmall cell lung cancer: Expression profiling of cancer-related genes in squamous cell lung cancer. Cancer Genet Cytogenet 149: 98-106, 2004.

25. Liau SS, Jazag A and Whang EE: HMGA1 is a determinant of cellular invasiveness and in vivo metastatic potential in pancreatic adenocarcinoma. Cancer Res 66: 11613-11622, 2006.

26. Yuan S, Pan Q, Fu C and Bi Z: Silencing of HMGA1 expression by RNA interference suppresses growth of osteogenic sarcoma. Mol Cell Biochem 355: 281-287, 2011. 